\title{
Adrenomedullin prevents apoptosis in prostate cancer cells
}

\author{
Ibane Abasolo, Luis M. Montuenga, Alfonso Calvo* \\ Division of Oncology, Center for Applied Medical Research (CIMA) and Department of Histology and Pathology, University of Navarra, Pamplona, Spain
}

Received 26 July 2005; accepted 22 September 2005

Available online 17 November 2005

\begin{abstract}
The 52-aminoacid peptide adrenomedullin (AM) is expressed in the normal and malignant prostate. We have previously shown that prostate cancer cells produce and secrete AM, which acts as an autocrine growth inhibitory factor. We have evaluated in the present study the role of AM in prostate cancer cell apoptosis, induced either by serum deprivation or treatment with the chemotherapeutic agent etoposide (which acts as an inhibitor of topoisomerase II). For this purpose we over-expressed AM in PC-3, DU 145 and LNCaP cells, which were transfected with an expression vector carrying AM. We also treated the parental cell lines with synthetic AM in normal culture conditions and in conditions of induced-apoptosis. After serum removal, AM prevented apoptosis in DU 145 and PC-3 cells, but not in LNCaP cells. When treated with etoposide, AM prevented apoptosis in PC-3 and LNCaP cells, but not in DU 145 cells. Cell cycle analysis demonstrated a significant decrease in the percentage of AM-overexpressing PC-3 cells in the subG0/G1 phase after treatment with etoposide, as compared to the percentage of mocktransfected PC-3 treated cells. Western blot showed that protein levels of phosphorylated ERK1/2 increased in parental PC-3 cells after treatment with etoposide. In PC-3 cells overexpressing AM, phosphorylated ERK1/2 basal levels were lower than basal levels of parental PC-3 cells, and treatment with etoposide did not result in such an increase. Etoposide produced a significant increase in cleaved PARP in parental PC-3 cells. However, PC-3 clones overexpressing AM that were treated with etoposide only showed a mild increase in fragmented PARP. The ratio Bcl-2/Bax was reduced in parental or mock-transfected PC-3 cells after treatment with etoposide. On the contrary, this ratio was not reduced in PC-3 clones with AM overexpression that were treated with etoposide. All these data demonstrate that AM plays a protective role against induced apoptosis in prostate cancer cells. These results may have important implications in prostate cancer resistance to chemotherapeutic agents.
\end{abstract}

(C) 2005 Elsevier B.V. All rights reserved.

Keywords: Adrenomedullin; Apoptosis; Bcl-2; Bax

\section{Introduction}

Prostate cancer $(\mathrm{PrCa})$ is a leading cause of cancer mortality among men in western countries [1]. The incidence of PrCa has increased during the last decade, due in part to aging of the male population and the widespread use of screening tests [2]. Even though localized prostate cancer can be treated and cured, there is no successful treatment for the advanced androgenindependent metastatic disease [2]. Understanding molecular mechanisms involved in androgen refractory tumor growth is a hallmark of prostate cancer research. The finding of genes whose expression is altered in androgen-independent prostate

\footnotetext{
* Corresponding author. Laboratory 1.05 Division of Oncology CIMA, University of Navarra Pio XII, 55, 31008, Pamplona, Spain. Tel.: +34 948 194700x1011; fax: +34 948194714 .

E-mail address: acalvo@unav.es (A. Calvo).
}

cancer will provide additional targets for therapy. In this regard, high throughput microarray analyses are currently providing new gene candidates for such a purpose [3]. It has been suggested that growth factors which regulate locally cell-cell interactions, could play a key role in tumor growth after androgen withdrawal [4]. We and others have previously reported that adrenomedullin (AM), a 52-aminoacid peptide isolated from a pheochromocytoma [5], is highly expressed in the normal and malignant prostate [6-8]. Expression of AM in LNCaP cells increases when androgens are removed from the culture medium [9]. Our previous experiments in vivo using androgen-dependent prostate tumors xenografted into nude mice showed that the expression of AM increases after castration [9]. Rocchi et al. [10] have shown an increase in AM levels in LuCaP 23.1 xenografted tumors after castration.

Numerous roles have been attributed to AM in different organs, such as vasodilation, bronchodilation, hormone secre- 
tion control, and regulation of cell growth [11]. We have recently shown that AM causes cell growth inhibition in PC-3 and $\mathrm{LNCaP}$ prostate cancer cells and decreases tumor growth in vivo in xenograft models $[12,13]$. We have also demonstrated that overexpression of AM in PC-3 cells changes the transcriptional program of approximately 100 genes compared to the parental cells, including GADD45, IFG-BP6, RUNX3, IL-13R $\alpha 2$, IL-8, and tenascin C [14]. A group of 10 dysregulated genes were related to apoptosis, whereas other genes were related to cell adhesion, extracellular matrix proteins, and tumor-related cytokines [14].

Three receptors for AM have been described: L1, RDC1, and the calcitonin receptor-like receptor (CL) coupled with the receptor activity modifying proteins (RAMPs) [11]. Among these, the CL/RAMPs complex seems to be the most important one. CL requires the presence of RAMPs for intracellular signaling [15]. RAMP-1 acts as a calcitonin gene related protein (CRGP) and AM receptor when coupled with CL. However, it binds with much more affinity to CGRP than to AM [15]. RAMP-2 or RAMP-3 coupled with CL bind specifically to AM [15]. PC-3, LNCaP and DU 145 express the CL/RAMP-2 receptor complex [13], and AM acts as an autocrine factor for these cells [13].

Several studies have shown that AM provides cells protection from undergoing apoptosis. Such a result has been demonstrated in breast cancer [16], endometrial cancer [17], and endothelial [18] cells. Culture of cells under stressful conditions, such as exposure to TNF $\alpha$ [19], hypoxia [17] or serum deprivation $[20,18]$ stimulates AM expression. Since our previous publications showed that $\mathrm{AM}$ causes cell cycle arrest and subsequent cell growth inhibition in PC-3 and LNCaP cells, we hypothesized in the present study that AM would favor cells to undergo induced-apoptosis. Our results turned out to be opposite to this hypothesis. PC-3 or LNCaP cells treated with AM plus etoposide, or cultured without serum plus AM had a survival advantage with respect to untreated cells. The same result was found for cells overexpressing AM. Moreover, proteins of the apoptotic cascade were not as induced in AM-overexpressing cells as in the parental cells. We suggest that AM can have a dual role in prostate cancer cells regulating differently cell growth (depending upon stress culture conditions), and that AM can play a role against resistance to chemotherapeutic drugs.

\section{Materials and methods}

\subsection{Cell culture}

Prostate cancer cell lines (PC-3, DU 145 and LNCaP) were cultured in RPMI 1640 medium with Glutamax-I (Invitrogen, Paisley, UK) supplemented with $10 \%$ fetal calf serum (FCS; Invitrogen), $100 \mathrm{U} / \mathrm{mL}$ penicillin and $100 \mu \mathrm{g} / \mathrm{mL}$ streptomycin (Invitrogen) (complete medium). PC-3 and DU 145 cell clones overexpressing $\mathrm{AM}$ were obtained by stable transfection of the pcDNA3.1/Hygro/LacZ plasmid, and have been previously established and characterized by our group $[12,13]$. Clones were grown in the complete medium plus $150 \mu \mathrm{g} / \mathrm{mL}$ hygromycin B (Invitrogen) for selection of the plasmidcontaining cells. For LNCaP cells, transient transfections were used, since stably transfected clones lost expression of AM after 1 week in culture [13].

\subsection{MTT and cell cycle assays}

Two methods for the induction of apoptosis were used: (a) Culture of cells in serum-removed medium and (b) treatment with etoposide (Sigma, Barcelona, Spain). Etoposide was diluted in DMSO, so that the final concentration of DMSO was less than $1 \%$ of the culture medium volume. For MTT assays in serum-deprived conditions, $2.4 \times 10^{4}$ cells/well were plated in 96-well plates, in RPMI without FCS for 0, 24, 48 and $72 \mathrm{~h}$. For MTT assays with etoposide, the same number of cells was plated in RPMI with $10 \%$ FCS.

To test the effect of AM on cell proliferation, hAM (Peninsula-Bachem, San Carlos, CA) was added daily to the cell culture medium at a final concentration of $100 \mathrm{nM}$. Treatment with etoposide was applied to the cells $24 \mathrm{~h}$ after plating, at a concentration of $100 \mathrm{mg} / \mathrm{L}$.

For cell cytometry analysis, synchronized cells were grown for $24 \mathrm{~h}$ in complete medium, and then treated or untreated with $100 \mathrm{mg} / \mathrm{L}$ etoposide for 24,48 and $72 \mathrm{~h}$. Cells were then collected and processed with the DNA Prep Coulter kit (Beckman Coulter, Fullerton, CA), following the manufacturer's instructions. Cells were analyzed in a FacsCalibur flow cytometer (Becton Dickinson) with a $488 \mathrm{~nm}$ excitation wavelength.

\subsection{Western blot}

Protein extraction from cell lysates was performed in RIPA buffer when antibodies anti-Bcl-2, -Bax, -PARP, and - $\beta$ actin were used. For phosphorylated and total ERK1/2 detection, the lysis buffer consisted of: $25 \mathrm{mM}$ sodium $\beta$-glycerolphosphate, $1 \mathrm{mM}$ sodium orthovanadate, $25 \mathrm{mM} \mathrm{NaF}, 1 \mathrm{mM} \mathrm{Na}_{2} \mathrm{P}_{3} \mathrm{O}_{4}, 1 \%$ TritonX-100, $0.05 \%$ sodium deoxycholate, $1 \mathrm{mM}$ benzamidine, $10 \%$ glycerol, $0.1 \%$-mercaptoethanol, $0.01 \%$ SDS, $5 \mathrm{mM}$ EDTA, and 5 mM EGTA (all of them from Sigma, Steinheim, Germany). Both extraction buffers contained a cocktail of protease inhibitors (Complete ${ }^{\mathrm{TM}}$; Roche Diagnostics, Penzberg, Germany). Protein concentration was spectrophotometrically measured using the $\mathrm{BCA}^{\mathrm{TM}}$ protein assay kit (Pierce, Rockford, IL, USA). Sixty micrograms of protein were loaded in each well, which where separated by SDS-PAGE in $4-12 \%$ BisTris polyacrylamide pre-cast gels (Invitrogen). Tris-acetate gels (Invitrogen) were used to detect PARP. Electrophoresis was performed in the presence of 5\% 2- $\beta$-mercaptoethanol for 45 $\min$ at $200 \mathrm{~V}$. Proteins were transferred to a $0.22 \mu \mathrm{m}$ nitrocellulose membrane (BioRad, Hercules, CA) at $30 \mathrm{~V}$ for $1 \mathrm{~h}$, and membranes were stained with Ponçeau solution. Blots were then incubated for $2 \mathrm{~h}$ at RT in blocking solution (Tropix, Bedford, MA). Polyclonal anti-Bcl-2 and anti-Bax antibodies (both from Santa Cruz) were used at 1:500 and 1:1000 dilution, respectively. Polyclonal anti-PARP (Promega, Madison, WI) was used at 1:5000 and monoclonal anti- $\beta$ actin antibody 

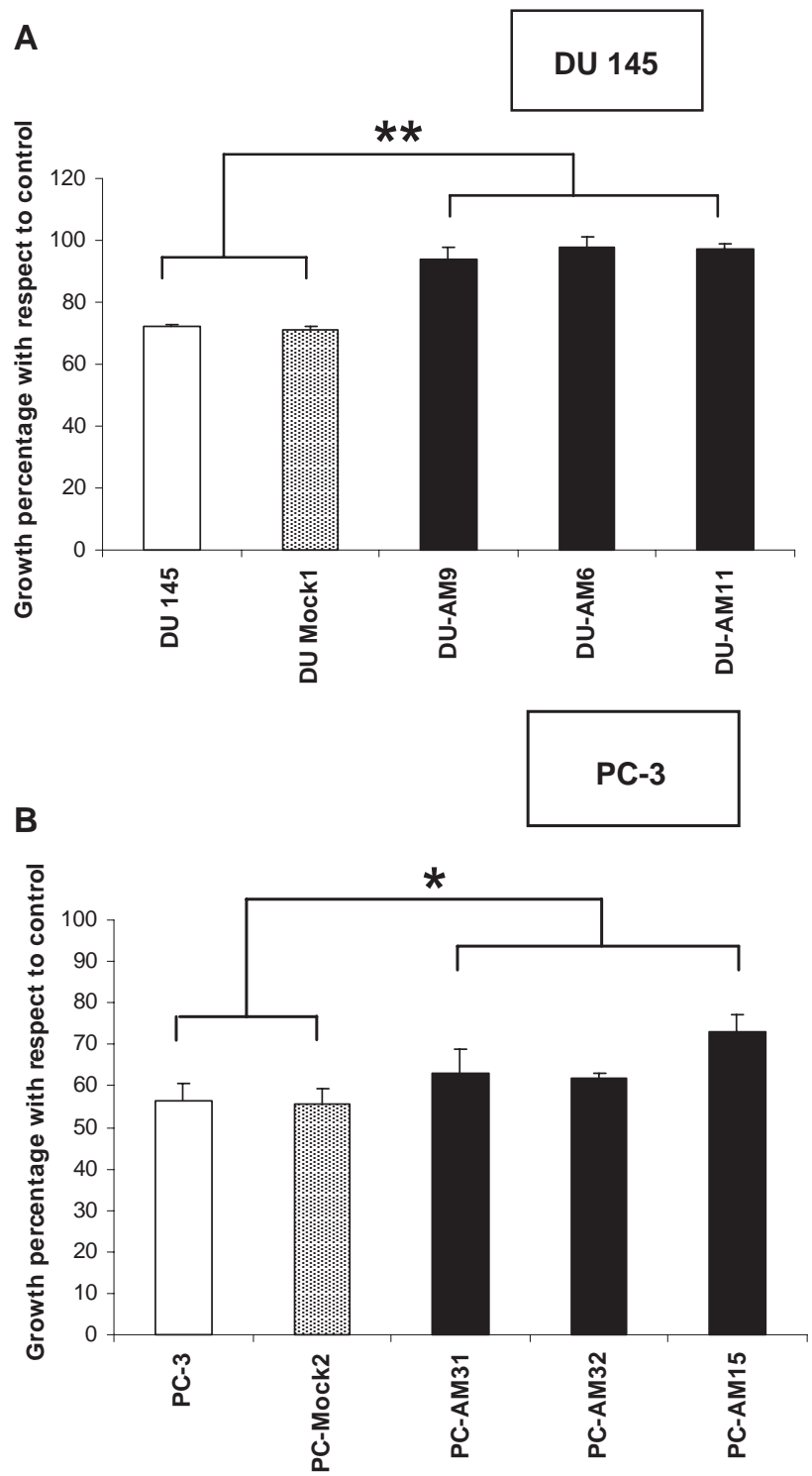

Fig. 1. MTT assays of DU 145 (A) and PC-3 (B) prostate cell clones $24 \mathrm{~h}$ after serum removal. A: DU 145 cell clones that overexpress AM (DU-AM9, DUAM6, and DU-AM11) show a significant increase in proliferation rates (**, $p<0.001$ ) compared to control cells (DU 145 parental and DU-145 Mock1 cells). B: PC-3 clones with AM overexpression (PC-AM31, PC-AM32, and PC-AM15) also show a significant growth advantage $(*, p<0.05)$ with respect to controls (PC-3, and PC-Mock2).

(Sigma) at 1:10000. For ERK1/2 detection, antisera to detect phosporylated and total ERK1/2 (Cell Signaling, Beverly, MA) were diluted at 1:1000. Incubation with the appropriate secondary antibody and chemoluminiscence detection kit (Tropix) were used for visualization of the signal.

\section{Results}

\subsection{AM protects prostate cancer cells from apoptosis-induced cell death}

Two widely used methods to induce apoptosis in prostate cancer cells are the depletion of serum in the culture medium and treatment with etoposide. To evaluate whether AM was involved in prostate cancer cell apoptosis we used cell clones that overexpress AM, or exogenous treatment with the AM peptide. We have previously generated and characterized PC-3 (AM15, AM32, AM31), DU 145 (AM6, AM9, AM11), and LNCaP (LNCaP-AM) cells with high or intermediate expression of AM, and mock-transfected clones (Mock-2 for PC-3, Mock-1 for DU 145, Mock-LNCaP for LNCaP cells) [13]. First, we compared cell viability of parental, mock-transfected or AM-transfected clones in serum-deprived culture media. Twenty-four hours after serum removal, cell viability was significantly higher in DU 145 and PC-3 cell clones that overexpressed AM, compared to the parental or mocktransfected cells (Fig. 1). In LNCaP cells, however, overexpression of AM did not modify cell viability when compared
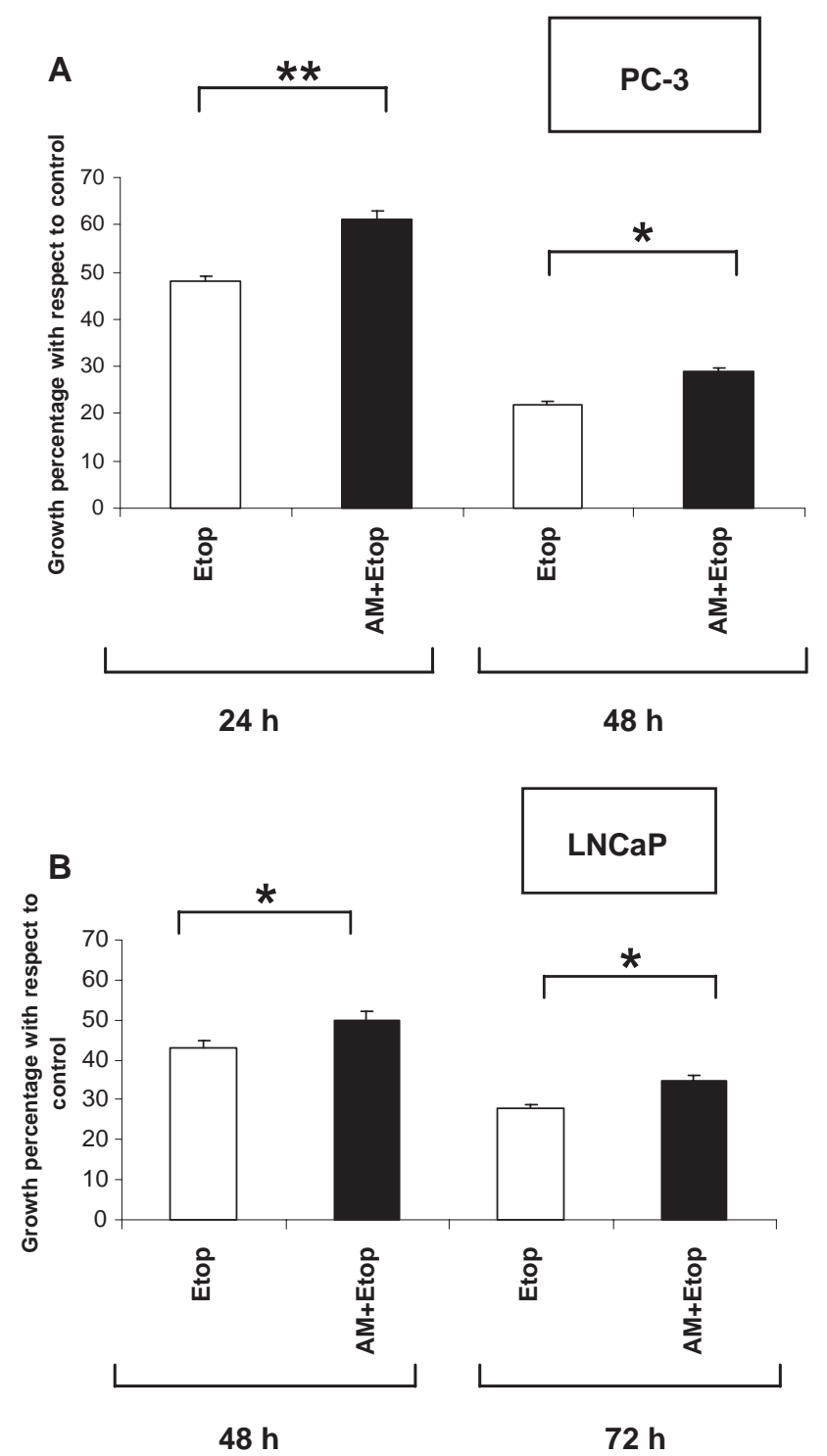

Fig. 2. MTT assays of PC-3 (A) and LNCaP (B) prostate cells after treatment with etoposide, or etoposide plus AM. A: The percentage of viable PC-3 cells $24 \mathrm{~h}$ and $48 \mathrm{~h}$ after treatment with etoposide is significantly higher when cells are treated with AM. B: A similar result is found for $\mathrm{LNCaP}$ cells. Exposure to AM plus etoposide provides a significant growth advantage $(p<0.05)$ over cells treated with etoposide alone. ${ }^{* *} p<0.01 ;{ }^{*} p<0.05$. 


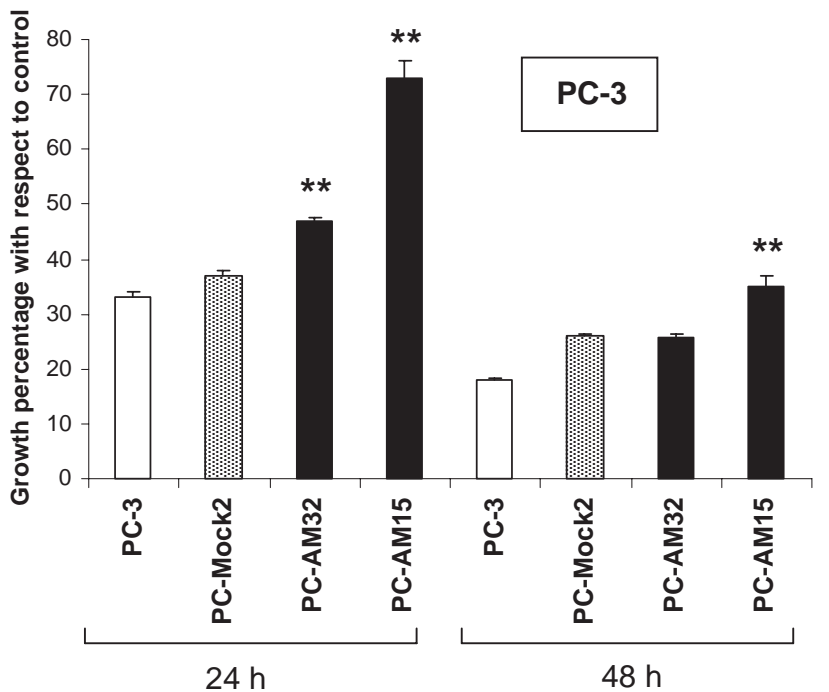

Fig. 3. Cell survival in PC-3 clones treated with etoposide for $24 \mathrm{~h}$ or $48 \mathrm{~h}$. PC3 cell clones with high expression of AM (PC-AM32, and PC-AM15) show significantly higher survival rates than controls (PC-3, and PC-Mock2). $* * p<0.01$.

to controls (either parental or mock-transfected cells) (result not shown).

Cell viability was also assessed in parental prostate cancer cells treated with etoposide, or etoposide plus AM peptide. Treatment with AM significantly prevented etoposide-induced apoptosis in PC-3 and LNCaP cells (Fig. 2), but had no effect in DU 145 cells (result not shown). The protective effect was especially significant in PC-3 cells, whereas LNCaP cells showed a milder cell growth advantage (Fig. 2). Parental PC-3, mock-transfected and AM-overexpressing clones were also treated with etoposide and tested for cell viability. Clones PCAM32 and PC-AM15, which expressed high levels of AM showed a significant increase in cell viability as compared to the parental or mock-transfected cells (Fig. 3).

We next conducted cell cycle analysis in PC-3 clones. The percentage of cells in the subG0/G1 phase of the cell cycle (which suggests apoptosis) was quantified in PC-3, mocktransfected, and clones overexpressing AM (PC-AM31, and PC-AM32), which were previously treated or untreated with etoposide. Overexpression of AM resulted in a significant decrease in the percentage of cells in the subG0/G1 phase (Fig. 4).

\subsection{AM increases the Bcl-2/Bax ratio and reduces phosphor- ylated ERK1/2 levels in etoposide-treated cells}

Because of the importance of etoposide as a chemotherapeutic drug frequently used in patients with androgenindependent prostate cancer, we further analyzed mechanisms of AM-mediated protection against etoposide-induced apoptosis. PC-3 cells were selected for these assays because of their androgen independence and for being more sensitive to $\mathrm{AM}$ than DU 145 and LNCaP cells.

Intracellular levels of phosphorylated ERK1/2 (p-ERK1/2) usually correlate with cell proliferation and survival [21].
However, treatment of cancer cells with etoposide or other genotoxic substances can also induce p-ERK1/2 [22,23]. We have previously shown that overexpression of AM causes decrease in p-ERK1/2 in PC-3 cells [13]. Treatment of parental or mock-transfected PC-3 cells with etoposide resulted in an increase in p-ERK1/2 without change in total ERK1/2 levels (Fig. 5). In PC-3 clones with overexpression of $\mathrm{AM}$, as mentioned before, there was a decrease in p-ERK $1 / 2$ without etoposide treatment. After incubation with etoposide, a mild increase in p-ERK1/2 levels was observed in AM-overexpressing clones. Nonetheless, such an increase was not as strong as the increase found for the controls (Fig. 5). These results show
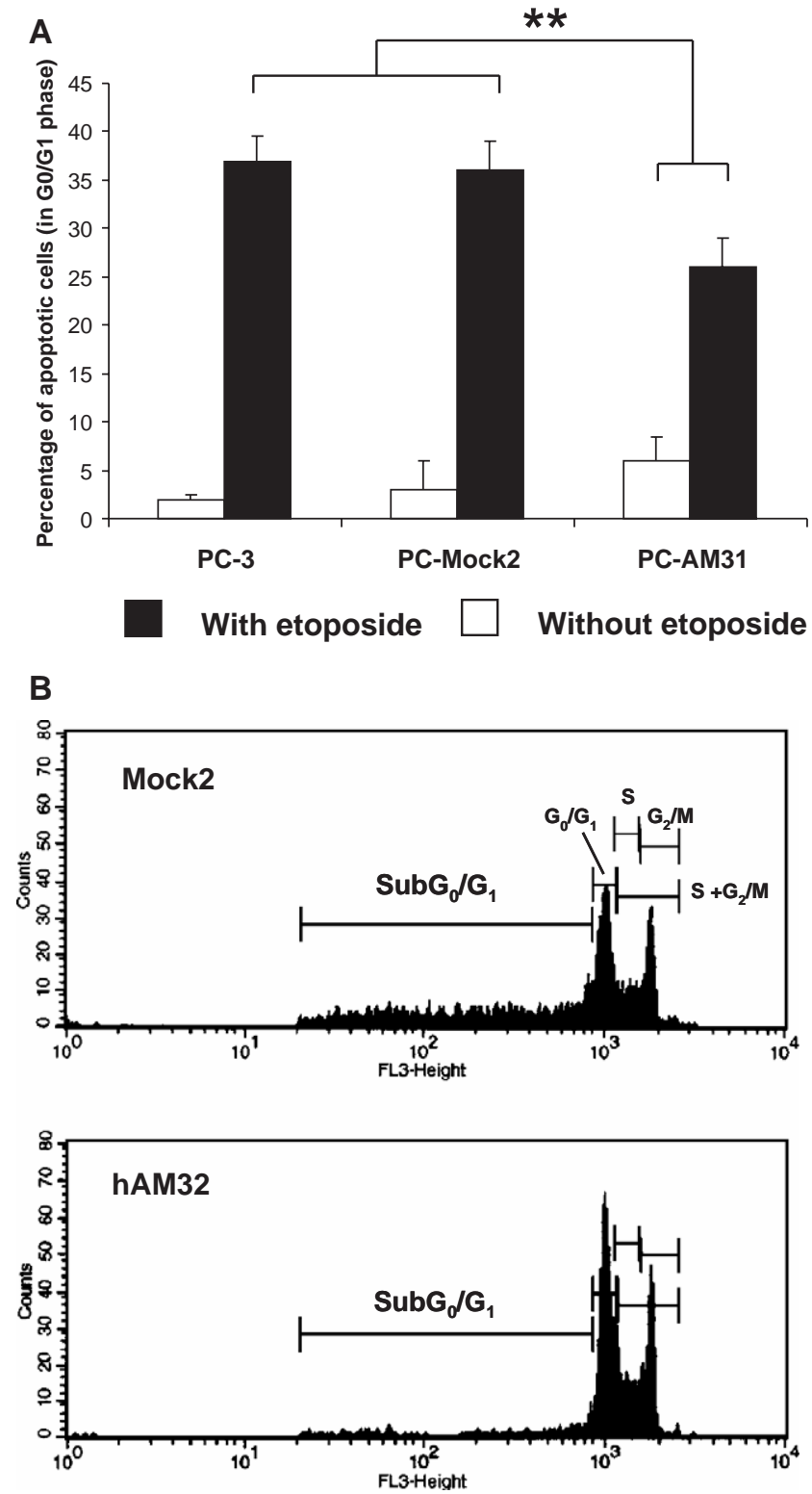

Fig. 4. Cell cycle analysis in PC-3 clones treated or untreated with etoposide. The percentage of cells in the subG0/G1 phase (suggesting apoptosis) increases dramatically when cells are treated with etoposide, irrespective of AM levels. However, in clones overexpressing AM (PC-AM31 for (A); PC-AM32 for (B)), such an increase is significantly lower $(* * p<0.01)$ than that found for control cells (PC-3, and PC-Mock2), after treatment with this chemotherapeutic agent. 


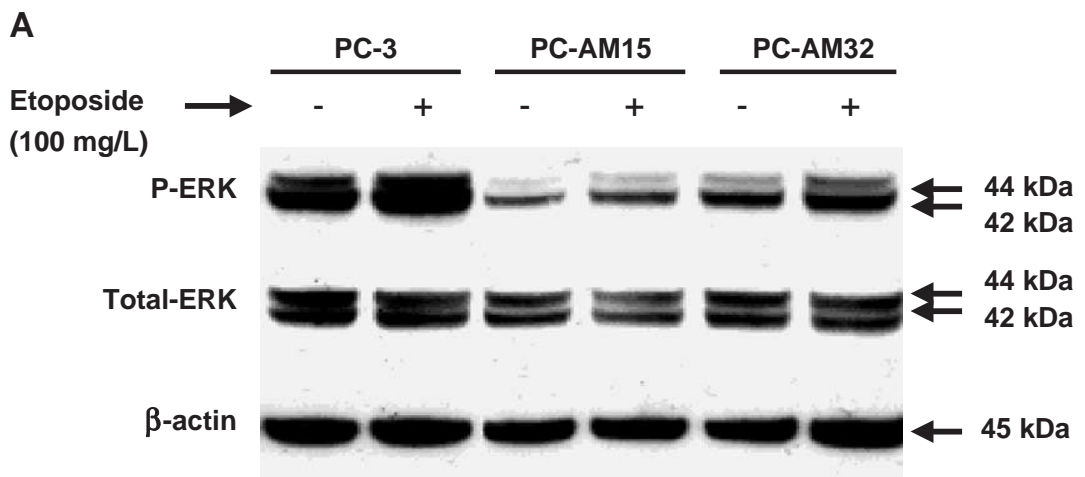

B

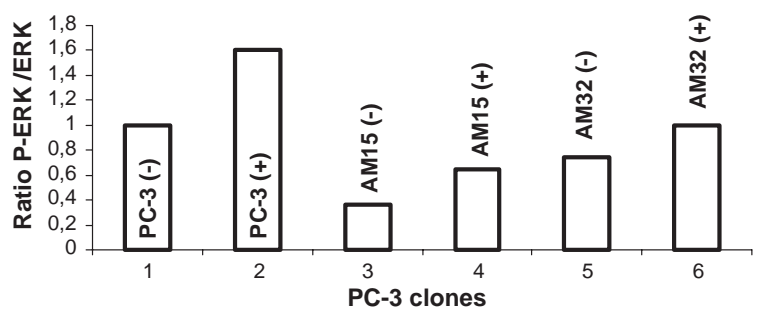

Fig. 5. Effect of AM on the induction of p-ERK1/2, after treatment with etoposide. A: Parental cells treated with etoposide show an increase in p-ERK1/2 levels with no change in total ERK1/2 levels. PC-3 clones with AM-overexpression (PC-AM15, and PC-AM32) have very low levels of p-ERK, as compared to parental cells. When PC-AM15 or PC-AM32 clones are treated with etoposide, the induction of p-ERK1/2 levels is not as pronounced as the increase that was observed for the parental cells. B: Densitometric quantification of the bands, normalization with $\beta$-actin, and calculation of the ratio p-ERK1/2/Total ERK1/2 confirmed the results.

that etoposide-mediated p-ERK1/2 induction is impaired in cells with high expression of AM.

The apoptotic effect of etoposide on PC-3 cells was analyzed by cleaved PARP, Bcl-2 and Bax Western blotting (Fig. 6). In control cells, a band of $85 \mathrm{kDa}$, corresponding to fragmented PARP was found after treatment with etoposide (Fig. 6). No signal was observed in untreated cells. In PC-3 clones with high expression of AM (PC-AM15 and PCAM32), the $85 \mathrm{kDa}$ band was also found after exposure to etoposide, but the intensity of the bands were significantly weaker than that of controls (Fig. 6). This result shows that, indeed, AM protects cells from induced apoptosis.

Bcl-2 protein was constitutively expressed in PC-3 cells. Levels of Bcl-2 were decreased in parental PC-3 cells after treatment with etoposide (Fig. 6). On the contrary, Bax was upregulated after incubation with etoposide (Fig. 6). Therefore, the $\mathrm{Bcl}-2 / \mathrm{Bax}$ ratio in $\mathrm{PC}-3$ cells treated with etoposide decreased as compared to the ratio observed for untreated parental cells. A similar result was observed for PC-3 cells transfected with the empty vector (PC-Mock2) (Fig. 6). When the AM-overexpressing PC-3AM15 and PC-AM32 clones were treated with etoposide, no such a reduction of $\mathrm{Bcl}-2$ nor induction of Bax were observed (Fig. 6). Therefore, the Bcl-2/ Bax ratio remains high, as opposed to the parental or mocktransfected cells (Fig. 6). These results show protection against pro-apoptotic signalling in PC-3 cells overexpressing AM.

\section{Discussion}

A previous study from our group showed that adrenomedullin causes anti-mitogenic effects and cell cycle growth arrest in PC-3 and LNCaP prostate cancer cells, when cells are grown in serum-containing culture medium [13]. The same result was found when AM levels were kept constantly elevated after stable transfection of an expression vector. Moreover, tumor volume was significantly reduced (by 50\%) in nude mice injected with PC-3 clones overexpressing AM, compared to controls (either the parental cells or cells stably transfected with the empty plasmid). Adrenomedullin seemed to act through an autocrine loop in these cells to produce such an effect, since they express both $\mathrm{AM}$ and the $\mathrm{AM}$ receptor complex $\mathrm{CL} /$ RAMP-2 [13].

We have previously shown as well by microarray analyses that overexpression of AM in PC-3 cells leads to changes in the transcriptional program of approximately 100 genes, among which there were many related to cell cycle arrest and apoptosis [14]. AM overexpression resulted in increased mRNA levels of GADD45A, IGF-BP6, STK-17, Bcl-2-related protein A1, 5'-nucleotidase (CD-73), TNF- $\alpha$ induced protein, and RUNX3 transcripts. These results are in agreement with the cell inhibitory effect that we described previously. In view of these results, we hypothesized in the present study that AM could sensitize prostate cells to undergo apoptosis. Our work here shows an opposite result than we expected. Indeed, prostate cancer cells that overexpress $\mathrm{AM}$, or are treated exogenously with an AM peptide are more refractory to undergo apoptosis than control cells.

The first method used to induce programmed cell death was the removal of serum from the culture medium, which gets rid of growth factors and results in the inability of cells to pass through the G1 restriction point and entering in the cell cycle [24]. The second method consisted of treating the cells with etoposide, which is a topoisomerase II inhibitor $[25,26]$. We have found that AM protects prostate cancer cells from 


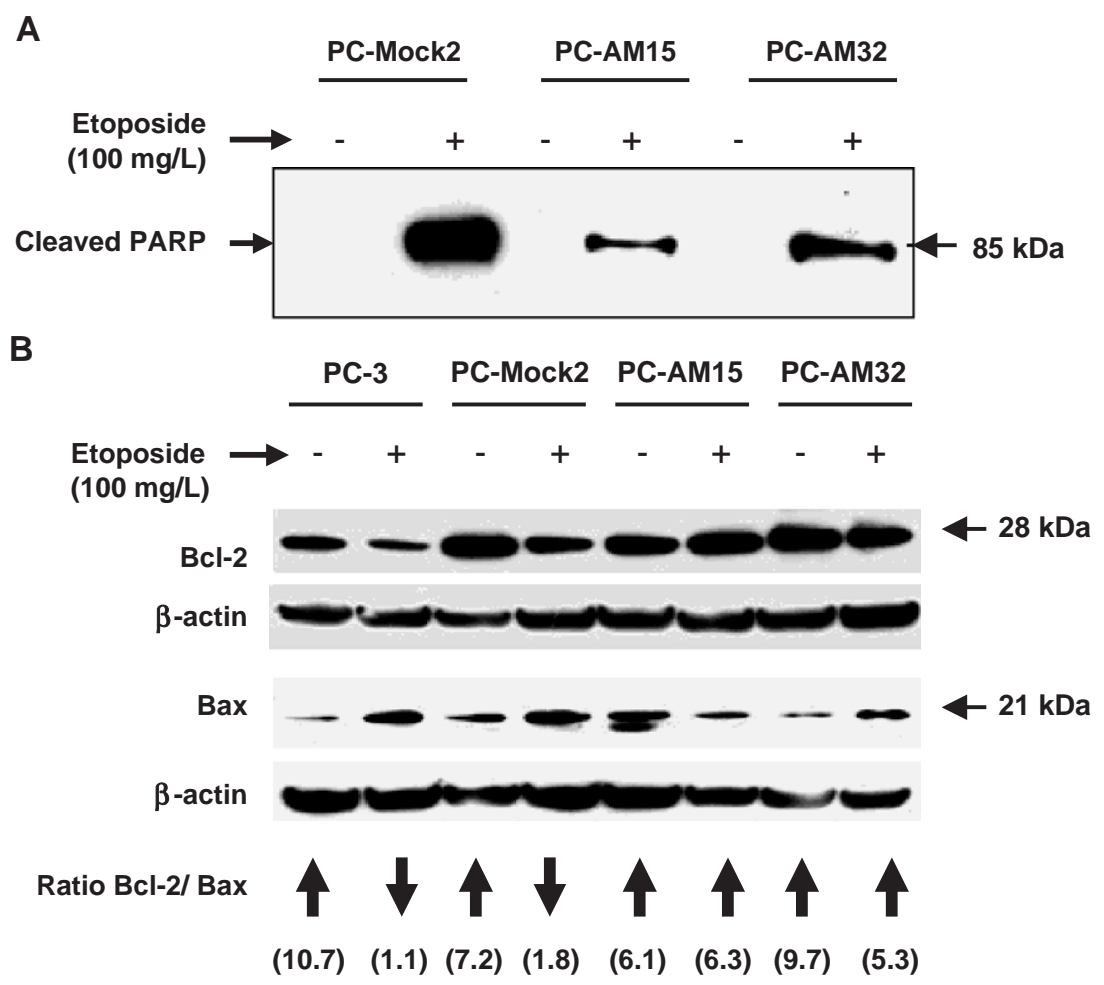

C

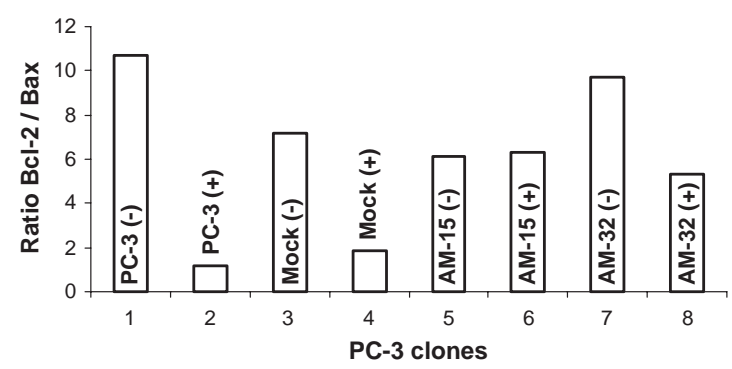

Fig. 6. Effect of AM on apoptosis. A: After exposure to etoposide, Western blot for cleaved PARP shows a strong signal in PC-3 mock-transfected cells (controls). PC-3 clones overexpressing AM (PC-AM15 and PC-AM32) show a less intense band. B: Bcl-2/Bax protein expression in PC-3 clones. Treatment of control cells (PC-3 or PC-Mock2) with etoposide leads to decrease in Bcl-2 and increase in Bax levels, as compared to untreated cells. On the contrary, clones with high expression of AM do not show such a dramatic change. C: Densitometric quantification of the bands, normalization with $\beta$-actin, and calculation of the ratio Bcl-2/ Bax. In control cells, Bcl-2/Bax ratio decreases after treatment with etoposide. However, such a decrease is not seen if cells overexpress AM.

undergoing apoptosis, the protective effect being different for each cell type depending on the method of induction. AM prevents apoptosis in DU 145 and PC-3 cells, but not in LNCaP cells, after serum deprivation. However, when apoptosis is induced by etoposide, AM is a protective factor for PC-3 and LNCaP, but not for DU 145 cells. Thus, PC-3 cells seem to be the type of cell line of choice to study the protective role of $\mathrm{AM}$ in prostate cancer. We have previously found in cell growth experiments that, unlike DU 145 cells, both PC-3 and LNCaP cells respond similarly to AM when cells are cultured in serum-enriched medium [13]. Dysruption of different genetic pathways in these cells are likely to be responsible for changes in cell growth and apoptosis responses after exposure to AM. LNCaP cells have a wild type p53 and are androgen sensitive; PC-3 cells are negative for p53 and are androgen independent; DU 145 cells have a mutated p53, are negative for Bax, and are insensitive to androgens as well [27]. We have shown here that etoposide causes increase in Bax levels in parental PC-3 cells, but not in AM-overexpressing cells. The functional alteration of Bax in DU 145 cells [28] can explain why these cells are not protected by AM when treated with etoposide.

Other studies have shown that AM plays a role in resistance to induced apoptosis. Martinez et al. [29] demonstrated that breast cancer cells that overexpressed AM are significantly less prone to die through apoptosis, when serum is removed from the culture medium. Resistance to apoptosis is related in these cells to a decrease in the pro-apoptotic molecules Bax and BID, and several caspases [29]. AM exerts an anti-apoptotic effect on endothelial cells cultured in serum-free conditions [18]. Such an effect occurs via cGMP- and Bcl-independent mechanisms [18]. Culture of endometrial cancer cells under hypoxic conditions leads to programmed cell death, which is prevented if cells are treated with or overexpress AM [17]. Bcl2 expression is induced more than 6-fold in these cells as a consequence of AM treatment, regardless of whether the cells 
are cultured under hypoxia or normoxia conditions [17]. Bcl-2 and AM are co-expressed in cervical cancer [30]. Importantly, in an in vivo rat model of ischemia and reperfusion, AM attenuates myocardial infarction and apoptosis [31]. This effect is caused through increase in Akt and Bad phosphorylation and Bcl-2 levels [31]. Our present results in prostate cancer cells also demonstrate that AM induces the expression of Bcl-2 and decreases Bax, which favours the anti-apoptotic signaling and prevents the cells from undergoing apoptosis.

Etoposide inhibits the activity of topoisomerase II thus causing G2/M cell growth arrest and apoptosis [25]. Exposure to etoposide results in double-stranded degradation of DNA, which triggers the apoptotic cascade $[25,26]$. Although the activation of ERK is usually related to cell survival and proliferation, drugs that induce apoptosis can also produce ERK activation $[22,23]$. The MAPK pathways (including ERK, JNK and p38) have been reported to be activated by genotoxic substances in many cell types [23,32]. Etoposide causes ERK1/ 2 activation in U937 leukemia cells [22]. We have found in the present study that levels of phosphorylated ERK1/2 increase in parental PC-3 cells after treatment with etoposide. In PC-3 cells overexpressing AM, phosphorylated ERK1/2 levels were lower than levels of parental PC-3 cells, and treatment with etoposide did not result in such an increase. This result also supports that the genotoxic effect of etoposide is less effective if cells have high expression of AM.

Taken together, all these data show that AM confers resistance to agents that induce apoptosis, involving the Bclfamily related signaling. In addition, AM expression is induced by HIF-1 $\alpha$ [33]. It is known that hypoxic areas of tumors are refractory to radiation and chemotherapy [34]. In this regard, AM could represent a putative tumor marker to predict response to radio- or chemotherapy, although this possibility has not been yet tested. AM also promotes tumor angiogenesis and enhances VEGF [35], and MMP-2 [36] levels, and is highly expressed in hypoxic tumors $[37,38]$. In renal cell carcinoma cells, hypoxia leads to AM and VEGF expression, which act in a coordinate way to facilitate cell growth [37]. All this could have therapeutical implications in cancer treatment, since it seems clear from different studies that AM provides resistance to stress and induced-cell death. Although more confirmatory experiments are needed, AM could represent a novel therapeutic target for hypoxic and radio-chemo-resistant tumors.

As mentioned before, our previous work demonstrated a cell growth inhibitory effect of AM in PC-3 and LNCaP cells cultured in non-stressful conditions. On the contrary, if these cells are subjected to stress (lack of serum or treatment with etoposide) AM provides a survival advantage. These results seem to be contradictory. However, it is possible that AM plays a dual role in the control of cell growth depending upon the external cell conditions. Other regulatory factors have demonstrated to play a dual role in either cell cycle promotion or induction of apoptosis, such as c-Myc [39]. This oncogene is a nuclear phosphoprotein that functions as a transcription factor stimulating both cell cycle progression and apoptosis. A "dual signal" model has been prostulated to explain the ability of cMyc to drive cell division or apoptosis [40,41]. However, the precise molecular mechanisms that induce these processes have not yet been identified. The conditions of the extracellular environment seem to be crucial in the function of c-Myc [41]. cMyc is activated by mitogenic signals to push cells into the G1 phase by activation of genes with a cell cycle promotion role and suppression of genes involved in cell growth arrest. AM and cMyc are indeed related genes. AM induces the expression of Max, a heterodimeric partner of c-Myc, which contributes to rescue endothelial cells to suffer apoptosis [20]. The formation of Max-Max homodimers suppresses apoptosis [42]. As Oehler et al. [17] pointed out, it is worth noticing that the anti-apoptotic proteins AM, Bcl-2, and Max interact with c-Myc.

In summary, we have demonstrated that AM provides a prostate cancer cell growth advantage against inducedapoptosis. Because of the relationship between AM and $\mathrm{Bcl}-2$, their high expression in hormone independent prostate cancer $[7,43]$, and the role of AM in hypoxia and angiogenesis [35], we suggest that AM should be taken into consideration as a target for therapy. Hormone refractory prostate cancer is often resistant to chemotherapeutic drugs, such as etoposide, and Bcl-2 plays a key role in blocking the pro-apoptotic cascades [44]. Clinical trials using etoposide are currently underway to treat androgen-independent prostate cancer. Designing strategies to sensitize prostate cancer cells to etoposide or other chemotherapeutic agents could substantially improve therapy. In this regard, inhibition of AM and Bcl-2 may be relevant for androgen-independent prostate cancer sensitization against chemotherapy.

\section{Acknowledgments}

We thank Raul Catena for helping us in the densitometric quantification of the Western blot bands. Ibane Abasolo was supported by a Basque Government predoctoral grant. This work was supported by a grant from the Spanish Government of Health, RTICCC 03/10.

\section{References}

[1] Greenlee RT, Murray T, Bolden S, Wingo PA. Cancer statistics, 2000. CA Cancer J Clin 2000;50(1):7-33.

[2] Wilson SS, Crawford ED. Screening for prostate cancer: current recommendations. Urol Clin North Am 2004;31(2):219-26.

[3] Glinsky GV, Glinskii AB, Stephenson AJ, Hoffman RM, Gerald WL. Gene expression profiling predicts clinical outcome of prostate cancer. J Clin Invest 2004;113(6):913-23.

[4] Culig Z, Hobisch A, Cronauer MV, Radmayr C, Hittmair A, Zhang J, et al. Regulation of prostatic growth and function by peptide growth factors. Prostate 1996;28(6):392-405.

[5] Kitamura K, Sakata J, Kangawa K, Kojima M, Matsuo H, Eto T. Cloning and characterization of cDNA encoding a precursor for human adrenomedullin. Biochem Biophys Res Commun 1993;194(2):720-5.

[6] Jiménez N, Calvo A, Martinez A, Rosell D, Cuttitta F, Montuenga LM. Expression of adrenomedullin and proadrenomedullin N-terminal 20 peptide in human and rat prostate. J Histochem Cytochem 1999;47(9): 1167-78.

[7] Rocchi P, Boudouresque F, Zamora AJ, Muracciole X, Lechevallier E, Martin PM, et al. Expression of adrenomedullin and peptide amidation activity in human prostate cancer and in human prostate cancer cell lines. Cancer Res 2001;61(3):1196-206. 
[8] Jiménez N, Jongsma J, Calvo A, van der Kwast TH, Treston AM, Cuttitta F, et al. Androgen-independent expression of adrenomedullin and peptidylglycine alpha-amidating monooxygenase in human prostatic carcinoma. Mol Carcinog 2003;38(1):14-24.

[9] Jimenez N, Abasolo I, Jongsma J, Calvo A, Garayoa M, van der Kwast TH, et al. Androgen-independent expression of adrenomedullin and peptidylglycine alpha-amidating monooxygenase in human prostatic carcinoma. Mol Carcinog 2003;38(1):14-24.

[10] Rocchi P, Muracciole X, Fina F, Mulholland DJ, Karsenty G, Palmari J, et al. Molecular analysis integrating different pathways associated with androgen-independent progression in $\mathrm{LuCaP} 23.1$ xenograft. Oncogene 2004;23(56):9111-9.

[11] Hinson JP, Kapas S, Smith DM. Adrenomedullin, a multifunctional regulatory peptide. Endocr Rev 2000;21(2):138-67.

[12] Abasolo I, Yang L, Haleem R, Xiao W, Pio R, Cuttitta F, et al. Overexpression of adrenomedullin gene markedly inhibits proliferation of PC3 prostate cancer cells in vitro and in vivo. Mol Cell Endocrinol 2003;199(1-2):179-87.

[13] Abasolo I, Wang Z, Montuenga LM, Calvo A. Adrenomedullin inhibits prostate cancer cell proliferation through a cAMP-independent autocrine mechanism. Biochem Biophys Res Commun 2004;322(3):878-86.

[14] Gonzalez-Moreno O, Calvo A, Joshi BH, Abasolo I, Leland P, Wang Z, et al. Gene expression profiling identifies IL-13 receptor alpha2 chain as a therapeutic target in prostate tumor cells overexpressing adrenomedullin. Int J Cancer 2004;17:17.

[15] McLatchie LM, Fraser NJ, Main MJ, Wise A, Brown J, Thompson N, et al. RAMPs regulate the transport and ligand specificity of the calcitonin-receptor-like receptor. Nature 1998;393(6683):333-9.

[16] Martinez A, Vos M, Guedez L, Kaur G, Chen Z, Garayoa M, et al. The effects of adrenomedullin overexpression in breast tumor cells. J Natl Cancer Inst 2002;94(16):1226-37.

[17] Oehler MK, Norbury C, Hague S, Rees MC, Bicknell R. Adrenomedullin inhibits hypoxic cell death by upregulation of Bcl-2 in endometrial cancer cells: a possible promotion mechanism for tumour growth. Oncogene 2001;20(23):2937-45.

[18] Sata M, Kakoki M, Nagata D, Nishimatsu H, Suzuki E, Aoyagi T, et al. Adrenomedullin and nitric oxide inhibit human endothelial cell apoptosis via a cyclic GMP-independent mechanism. Hypertension 2000;36(1):83-8.

[19] Hofbauer KH, Schoof E, Kurtz A, Sandner P. Inflammatory cytokines stimulate adrenomedullin expression through nitric oxide-dependent and -independent pathways. Hypertension 2002;39(1):161-7.

[20] Shichiri M, Kato H, Doi M, Marumo F, Hirata Y. Induction of max by adrenomedullin and calcitonin gene-related peptide antagonizes endothelial apoptosis. Mol Endocrinol 1999;13(8):1353-63.

[21] Guo C, Luttrell LM, Price DT. Mitogenic signaling in androgen sensitive and insensitive prostate cancer cell lines. J Urol 2000;163(3):1027-32.

[22] Jarvis WD, Johnson CR, Fornari FA, Park JS, Dent P, Grant S. Evidence that the apoptotic actions of etoposide are independent of c-Jun/activating protein-1-mediated transregulation. J Pharmacol Exp Ther 1999;290(3): 1384-92.

[23] Park SA, Park HJ, Lee BI, Ahn YH, Kim SU, Choi KS. Bcl-2 blocks cisplatin-induced apoptosis by suppression of ERK-mediated p53 accumulation in B104 cells. Brain Res Mol Brain Res 2001;93(1):18-26.

[24] Zetterberg A, Larsson O, Wiman KG. What is the restriction point? Curr Opin Cell Biol 1995;7(6):835-42.

[25] Hande KR. Etoposide: four decades of development of a topoisomerase II inhibitor. Eur J Cancer 1998;34(10):1514-21.

[26] Chen G, Shu J, Stacey DW. Oncogenic transformation potentiates apoptosis, S-phase arrest and stress-kinase activation by etoposide. Oncogene 1997;15(14):1643-51.
[27] Webber MM, Bello D, Quader S. Immortalized and tumorigenic adult human prostatic epithelial cell lines: characteristics and applications. Part 3. Oncogenes, suppressor genes, and applications. Prostate 1997; 30(2):136-42.

[28] Marcelli M, Marani M, Li X, Sturgis L, Haidacher SJ, Trial JA, et al Heterogeneous apoptotic responses of prostate cancer cell lines identify an association between sensitivity to staurosporine-induced apoptosis, expression of Bcl-2 family members, and caspase activation. Prostate 2000;42(4):260-73.

[29] Martinez A, Vos M, Guedez L, Kaur G, Chen Z, Garayoa M, et al. The effects of adrenomedullin overexpression in breast tumor cells. J Natl Cancer Inst 2002;94(16):1226-37.

[30] Li Z, Takeuchi S, Ohara N, Maruo T. Paradoxically abundant expression of Bcl-2 and adrenomedullin in invasive cervical squamous carcinoma. Int J Clin Oncol 2003;8(2):83-9.

[31] Kato K, Yin H, Agata J, Yoshida H, Chao L, Chao J. Adrenomedullin gene delivery attenuates myocardial infarction and apoptosis after ischemia and reperfusion. Am J Physiol Heart Circ Physiol 2003; 285(4):H1506-14 (Epub 2003 Jun 1512).

[32] Persons DL, Yazlovitskaya EM, Cui W, Pelling JC. Cisplatin-induced activation of mitogen-activated protein kinases in ovarian carcinoma cells: inhibition of extracellular signal-regulated kinase activity increases sensitivity to cisplatin. Clin Cancer Res 1999;5(5):1007-14.

[33] Garayoa M, Martinez A, Lee S, Pio R, An WG, Neckers L, et al. Hypoxia-inducible factor-1 (HIF-1) up-regulates adrenomedullin expression in human tumor cell lines during oxygen deprivation: a possible promotion mechanism of carcinogenesis. Mol Endocrinol 2000;14(6): $848-62$.

[34] Hockel M, Schlenger K, Hockel S, Vaupel P. Hypoxic cervical cancers with low apoptotic index are highly aggressive. Cancer Res 1999;59(18): $4525-8$.

[35] Iimuro S, Shindo T, Moriyama N, Amaki T, Niu P, Takeda N, et al. Angiogenic effects of adrenomedullin in ischemia and tumor growth. Circ Res 2004;95(4):415-23 (Epub 2004 Jul 2008).

[36] Tsuruda T, Kato J, Cao YN, Hatakeyama K, Masuyama H, Imamura T, et al. Adrenomedullin induces matrix metalloproteinase-2 activity in rat aortic adventitial fibroblasts. Biochem Biophys Res Commun 2004; 325(1):80-4.

[37] Fujita Y, Mimata H, Nasu N, Nomura T, Nomura Y, Nakagawa M. Involvement of adrenomedullin induced by hypoxia in angiogenesis in human renal cell carcinoma. Int J Urol 2002;9(6):285-95.

[38] Jogi A, Vallon-Christersson J, Holmquist L, Axelson H, Borg A, Pahlman S. Human neuroblastoma cells exposed to hypoxia: induction of genes associated with growth, survival, and aggressive behavior. Exp Cell Res 2004;295(2):469-87.

[39] Vermeulen K, Berneman ZN, Van Bockstaele DR. Cell cycle and apoptosis. Cell Prolif 2003;36(3):165-75.

[40] Pucci B, Kasten M, Giordano A. Cell cycle and apoptosis. Neoplasia 2000;2(4):291-9.

[41] Oster SK, Ho CS, Soucie EL, Penn LZ. The myc oncogene: marvelouslY complex. Adv Cancer Res 2002;84:81-154.

[42] Zhang H, Fan S, Prochownik EV. Distinct roles for MAX protein isoforms in proliferation and apoptosis. J Biol Chem 1997;272(28):17416-24.

[43] Kolar Z, Murray PG, Scott K, Harrison A, Vojtesek B, Dusek J. Relation of Bcl-2 expression to androgen receptor, p21WAF1/CIP1, and cyclin D1 status in prostate cancer. Mol Pathol 2000;53(1):15-8.

[44] Coffey RN, Watson RW, Hegarty PK, Watson CL, Wolohan L, Brady HR, et al. Priming prostate carcinoma cells for increased apoptosis is associated with up-regulation of the caspases. Cancer 2001;92(9):2297-308. 